\title{
Análisis del concepto de "posibilidad"
}

Si se entiende por progreso filosófico, no la solución definitiva de los grandes problemas tradicionales, sino la mayor penetración en el corazón de los mismos, el mejor esclarecimiento de sus conexiones, su más riguroso planteamiento y sobre todo el mayor avance en la vía asintótica de su solución, este trabajo tiene por objeto mostrar el gran progreso realizado por la Filosofía contemporánea respecto de algunos problemas fundamentales. Aunque según muchos detractores del pensamiento puro es esto imposible, la Filosofía progresa, avanza, se supera. Como un organismo pletóricôlde didac creces see expande, se ramifica, adquiere plenitud de forma.

No vamos a explicar el por qué profundo de este crecimiento. No deseamos efectuar un estudio del método moderno comparado con el antiguo, ni del alma objetiva que informa a la investigación filosófica moderna. Deseamos sencillamente exponer un ejemplo concreto, una muestra de los logros del pensamiento actual en relación a un problema céntrico de la Filosofía de todos los tiempos: el problema de la "Posibilidad". El lector que conozca aunque sea ligeramente el planteamiento antiguo, aristotélico-tomista, podrá apreciar el innegable avance que representa el tratamiento moderno, en tanto que comparado con el pri- 
mero. De esta manera podrá comprender mejor las semejanzas y las diferencias de las dos maneras de filosofar y podrá llegar a una visión general de su sentido y de su rol espiritual. Muchas veces se capta la esencia en forma más nítida y directa, mediante la contemplación abstractiva de lo concreto, que con el análisis de fórmulas sistematizadas y completamente abstractas.

El problema de la posibilidad ha sido siempre uno de los temas que con insistencia se han presentado en la temática filosófica de toda las épocas. Es sobre todo el fenómeno implacable del "devenir" lo que obliga a preguntar a todos los grandes pensadores clásicos por la posibilidad. El paso del no-ser al ser y del ser al no-ser, indica que es posible un hecho incomprensible. ¿Pero porqué es incomprensible?. Porque seguramente no se ha captado debidamente su posibilidad. Mas para vislumbrar esta posibilidad, hay que saber lo que es la posibilidad. Sólo sabiendo en qué consiste la "posibilidad" en general se puede saber cuando un algo es o no posible. Y empieza de esta manera el análisis del problema, analisis que tras milenario decurso debía de plasmarse en una de las adquisiciones más formidables del pensamiento contemporáneo.

Unicamente Aristóteles pudo entre los antiguos llegar a un planteamiento más o menos claro del problema. El problema es en sí tan hondo, tiene tantos visos de irracionalidad, que siempre ha sido un elemento perturbador en la sistematización filosófica. Platón, el divino Platón, no llegó jamás a un planteamiento claro del asunto. Por eso junto a las ideas, en tanto que explican la posibilidad de lo real, tiene que recurrir a un Demiurgo, extraño arquitecto que construye al mundo. 
Aristóteles se dió cuenta que la posibilidad era un concepto básico, sin cuyo esclarecimiento no se podía llegar a la elaboración de ningún sistema metafísico. Por eso toda su Filosofía no es en el fondo sino un esfuerzo por resolver el problema de la posibilidad. De alli el rol preponderante que otorga a la teoría de la Potencia y del Acto. La posibilidad de los sucesos del universo queda dilucidada en tanto que existe siempre una Potencia respecto de un Acto. Una cosa cualquiera está en potencia para devenir distinta de lo que fué. Está en potencia de adquirir una forma más perfecta, y cuando realiza esta forma, se dice que está en acto. La forma en si misma es la esencia, el eidos. Y en cuanto asumida por el ente anteriormente en potencia es la entelequia, el ente perfeccionado.

Toda potencia es susceptible de pasar al acto. La potencia tiende al acto, la materia tiende a la forma, lo inferior a lo superior. Así la posibilidad es esta tendencia de lo informe a lo formado, de lo indeterminado a lo determinado. Un suceso es posible, porque determinada cosa ha estado en potencia respecto de determinado acto, y al realizar esta potencia se há trasformado, originándose así una nueva cosa, relacionada con la anterior, pero más perfecta y acabada.

$\mathrm{Y}$ así en infinita procesión de potencias, se construye el universo, anhelo gigantesco de acto. Todo en él tiende al acto, $y$ al realizar esta tendencia se perfecciona. $Y$ de acto en acto se llega al acto puro, único ser que no está en potencia respecto de otro acto, único ser absolutamente perfecto, totalmente acabado, que cual cósmico imán regula el devenir universal.

Como casi todas las concepciones filosóficas de la antigüiedad, la teoría aristotélica es unilateral e imperfecta. 
Unilateral porque se refiere únicamente a la posibilidad biológica. El paso de la potencia al acto, la determinación de la materia por la forma, la definición de la posibilidad como "tendencia" hacia la forma, o como "disposición" de perfeccionamiento, son conceptos tomados todos del campo de lo vital. La relación cle la semilla con la planta ya logra. da, del animal pequeño con el ejemplar adulto, del niño con el hombre, son el subsuelo óntico sobre el que Aristóteles construyó su teoría. Los griegos no fueron sólo geómetras. También fueron biólogos. Por eso Platón explica el origen del universo por la acción de un Demiurgo, y Aristóteles reduce la posibilidad de todo acontecer a la disposición de perfeccionarse que posee todo ente. Esta disposición de evolucionar para adquirir determinada forma, es un factor estrictamente biológico. El mundo inorgánico no tiene nada que ver con ello. Los fenómenos son posibles en esta región del ser, no porque la materia bruta tenga tendencias latentes en su seno, sino sencillamente por la acción mecanica y ciega de las causas. Y asi mismo, la tendencia a devenir en acto que caracteriza a lo vital, no tiene nada que ver con la posibilidad de los pensamientos, o de las propiedades esenciales etc. Aristóteles captó determinada clase de posibilidad, y como la mayor parte de los metafísicos rebasó los límites posibles de la aplicación de su descubrimiento, y lo aplicó no únicamente a la vida como tal, sino a todo el universo.

Pero además de ser unilateral, su concepción es imperfecta, porque dentro del campo de lo biológico no basta aceptar la relación entre la potencia y el acto para determinar la posibilidad de los fenómenos vitales. ${ }_{7}^{\mathrm{La}}$ "dis- 
posición" es uno de los factores integrantes de la posibilidad biológica, pero no es el único.

El principal error de Aristóteles consiste en que no tuvo una visión general de la posibilidad. Creyó que la posibilidad de los objetos y de los sucesos, era algo interno a los mismos, algo fundamental dentro de sus más esenciales determinaciones. $Y$ sin embargo la posibilidad no es meramente algo interno. Puede ser también un factor externo.

Debido a las investigaciones de Kant, y de las escuelas neokantiana y fenomenológica, en la actualidad se ha llegado a una definición genérica de la posibilidad, aplicable a todas las regiones del ser, sin peligro de caer en la peligrosa unilateralidad en que cayeron los antiguos.

La posibilidad de un ente (entendemos por ente todo aquello que tiene ser, que es, ya sea en forma estática o dinámica) es el complejo de condiciones que determinan su existencia. La posibilidad no es así algo misterioso, oculto, interiór. Ess una arelación, Céntresla existencia de un ente y las condiciones cine là determinan.

La única dificultad que se encuentra en la definición, es el concepto de existencia. Es imposible precisarlo en forma rigurosa, pues es tan general, o casi tan general como el concepto de ser. Pero no es necesario definirlo rigurosamente para comprender la definición de posibilidad. Basta captarlo intuitivamente. Lo único que debe de hacerse, es evitar algunos malentendidos. Así por ejemplo, no hay que creer que con la palabra "existencia” se designa únicamente una propiedad de los entes reales, es decir de los entes individuales témporo-espaciales o meramente temporales. La palabra existencia designa a todo ente que tiene deter- 
minada subsistencia ontológica dentro de determinados grupos de entes. Puede por lo tanto hablarse de existencia ideal, de existencia lógica, de existencia noseológica. (Hartmann). Una esencia tiene que existir como esencia, pues de otra manera no sería esencia, no sería nada, no se podría captar como objeto de conocimiento. Desde luego no existe como ente real, en un tiempo y en un espacio. Pero existe como ente ideal en tanto que complejo de caracteres genéricos que determina los caracteres de cierta clase de entes reales. Así mismo un objeto cualquiera, puede no existir como objeto real o ideal, pero puede existir como objeto intencional, como objeto mentado por el sujeto cognoscente. La existencia es un factor ontológico generalísimo con múltiples especifícaciones. Hay existencia real (que fué la única que conocieron los antiguos) existencia ideal (de cuyo desconocimiento por la filosofía medioeval se derivaron una serie de falsos problemas como el de los tuniversales) existencia intencional (única distinta de la real conocidal por los-medioevales),-existencia lógica etc. Todas estas éspecies tienen de comun la subsistencia de determinado ente en medio de otros entes, su determinada "fuerza" ontológica, su resistencia a ser cambiado o destruído por actos mentales del sujeto (con excepción de la existencia intencional que es la más débil de todas las existencias). Pero desde luego que todas las especies de existencia se diferencian por el modo como poseen las anteriores propiedades. Así la existencia ideal se caracteriza por su gran subsistencia, ya que debido a su intemporalidad jamás un ente ideal deja de existir. El ente real deja cle existir después de determinado tiempo, pero en cambio tiene una enorme fuerza ontológica, se impone por sí mis- 
mo, obliga al sujeto conciente a "atender hacia él" (el caso de los fenómenos meteorológicos y telúricos). Ambas existencias no pueden ser destruídas por actos mentales del sujeto. La existencia intencional depende de la voluntad del sujeto, pero sin embargo una vez que ha sido planteada, es indestructible dentro del tiempo de planteamiento. Una vez creada, puede ser clestruída, pero ya nadie puede evitar que en determinado tiempo haya habido tal o cual existencia intencional. La existencia lógica tiene aún mayor subsistencia. que la ideal, y es una de las más generales, etc.

A la luz de la definición general anteriormente dada, vamos a examinar a las principales especies de posibilidad que se presentan en la abigarrada estructura del ser. El criterio para diferenciar las diferentes posibilidades se basa en la división del Ser en diversas regiones y esferas. Las principales esferas del Ser son dos: la real y la irreal. La esfera de lo real se divide en una serie de estratos intimamente compenetrados pero esencialnente difefentes. Entre los principales se pueden citavial estratosdel ser físico, al del ser biológico, al del ser anímico y al del ser espiritual. La esfera de lo irreal se divide en diversas subesferas o regiones. Entre las principales tenemos la región de lo ideal, la de lo lógico, la de lo noseológico y la de lo intencional.

Empezaremos por lo más fácil, es decir-aunque parezca paradójico-por la consiceración de la posibilidad en la esfera de lo irreal. Decimos que la teoría de la posibilidad de lo irreal es más sencilla que la teoría de la posibilidad de lo real, porque las condiciones que se necesitan para la existencia de los entes irreales son más sencillas y en menor número: 
La posibilidad del ente lógico consta de una sóla condición: la "no contradicción consigo mismo". Esto quiere decir que todo aquello que no es autocontradictorio posee existencia lógica: Así por ejemplo, la existencia de unos caballos alados es una existencia lógica, porque el concepto de alas, no contradice al concepto de caballo. Para que así fuera, alguno de los conceptos debería de contener entre sus notas, en forma explícita o implícita la negación del otro. Puede pues enunciarse el principio siguiente: "todo lo que no es autocontradictorio es lógicamente existente".

No hay que confundir a la posibilidad lógica con la ideal. Esta última es mucho más restringida y presupone a la primera. Todo lo que es posible idealmente es posible lógicamente, pero no al revés. La posibilidad ideal de un ente consta de dos condiciones: la ausencia de contradicción consigo mismo y la ausencia de contradicción con otros entes de la misma esfera. Esta segunda condición es lo que Leibniz con genial intuición llamó la "composibilidad".

Un ejemplo "aclara Ho dicho:iel teorema de Geometría que dice: la suma de los tres ángulos interiores de un triángulo es igual a $180^{\circ}$ vale únicamente dentro de los presupuestos de la Geometría euclideana. Pero si se aceptan los presupuestos de la Geometría elíptica, la suma de estos tres ángulos será menor de $180^{\circ}$, y si se acepta los postulados de la Geometría hiperbólica, la suma será mayor. En el campo dé estas geometrías la suma no puede ser $180^{\circ}$, porque se estaría contradiciendo los supuestos del punto de partida, es decir que no sería composible con la existencia de otros entes ideales.

Lógicamente este problema no tiene sentido. Lógica- 
mente no hay jamás variación de propiedades. $\mathrm{O}$ algo es contradictorio consigo mismo o no lo es. Y cuando no to es, existe lógicamente en forma eterna e indestructible. Por eso dijimos que la existencia lógica tenía aún una mayor subsistencia que la ideal.

La posibilidad intencional se subdivide en dos especies: la posibilidad de la intencionalidad imaginativa y la posibilidad de la intencionalidad de pensamiento. Ambas posibilidades son muy sencillas. La posibilidad de la intencionalidad imaginativa consta de dos condiciones: la éxistencia de la cualidad imaginada en la esfera de la percepción y la posibilidad lógica del objeto imaginado. La imaginación es un gran taller de combinación, es un caleidoscopio viviente y poderoso. Pero sólo puede combinar elementos. Crea relaciones pero no elementos. Las formas sensibles se dan a la imaginación, no son creadas por ellas. Por más genial que sea un artista jamás podrá imaginarse un color que no haya antes percibido o un sonido que no haya nunca escuchado. Lo único que puede hacer es relacionar los elementos percibidos en forma distinta y original. Pero estā originalidad etelacionalrtiene sus limites: no puede violar las leyes lógicas. Ningún artista, aunque su genialidad sea tan poderosa que raye en la locura, podrá jamás imaginarse un círculo cuadrado, o un triángulo re. dondo, ni un objeto distinto de sí mismo.

La posibilidad de la intencionalidad de pensamiento es mucho más sencilla que la de la intencionalidad imaginativa y únicamente consta de una condición generalísima: el sentido.

Todo aquello que tiene sentido puede pensarse y por lo tanto existe como objeto de pensamiento, es posible co- 
mo tal. Unicamente lo que no tiene sentido, lo que no se puede comprender como enunciado, carece de existencia intencional de pensamiento. Sólo el sinsentido queda fuera del campo universal del pensamiento. Fenómeno maravi1loso: de todas las posibilidades irreales, sólo la posibilidad del pensamiento no necesita de las condiciones de la posibilidad de los entes lógicos. Se puede pensar perfectamente un ente contradictorio consigo mismo, se puede comprender un enunciado sobre la existencia de dicho ente. Así si se dice "este círculo es cuadrado", "este triángulo es reclondo", "este hombre está vivo y está muerto", lo que se dice es absurdo, pero tiene un sentido perfectamente definido. Se comprende lo que se afirma, se sabe cual es el objeto de la mención intencional, se sabe cual es la meta del acto enunciativo. Por lo tanto objetos como "círculo cuadrado", "triángulo redondo" etc., pueden existir en la región del pensamiento, tienen posibilidad de pensamiento. Unicamente el sinsentido no puede existir en esta región, y por lo tanto en ninguna. Este grupo de letras: "Es ni grande la nota la anmiga: de Sócrates por " no constituye un enunciado, porque no puede ser comprendido. Es decirno tiene sentido, no se puede saber cual es el objeto hacia el cual apunta. Por lo tanto carece de toda posibilidad, es absolutamente imposible. El sinsentido es el sumum de la nimiedad, de la imposibilidad, no puede existir en ninguna forma.

De las consideraciones que anteceden se puede llegar a conclusiones curiosas. Por ejemplo, es completamente injusto el epíteto que daban los escolásticos a la imaginación al llamarla "la loca de la casa". La imaginación no tiene nada de loca. Puede ser poética, desbordante, hasta desor- 
denada. Pero es estrictamente lógica. Los principios de identidad, de contradicción, de tercio excluso son integramente respetados por ella. La verdadera "loca de la casa" o mejor el "loco de la casa" es el pensamiento, porque puede ser completamente ilógico y uno de los signos de la locura es el desconocimiento de los principios lógicos. Si se aplica lo dicho a la poesía, se puede considerar al surrealismo, dadaísmo etc., como un movimiento intelectualista. porque en ellos al aceptarse la contradicción y el absurdo, se excluye toda posibilidad de imaginación y sólo permanece el pensamiento. De manera, que aunque ilógicos, los poemas modernistas son intencionales, son ilaciones de puros pensamientos. Pero la consecuencia más importante es la grandiosidad del campode acción del pensamiento, su poder ilimitado para crear objetos. El pensamiento es siempre espontáneo, no está determinado por ninguna ley. Sólo se detiene ante una traba: el sinsentido. Pero ni la composibilidad de los conceptos, ni la ley universal deblosoprincipios lógiés łogran encerrarlo en marcos insalvabless. Elcpensaniento trasciende toda constricción, es lo único absolutamente libre y creador que tiene el hombre. No se puede imaginar colores o sonidos distintos, de los que se dan en la percepción, pero isí se pueden pensar! El pensamiento es absolutamente creador, porque no sólo puede crear las relaciones de los elementos, sino que también crea los elementos mismos. Es un caleidoscopio que a cada vuelta crea nuevas combinaciones y nuevos colores. Es debido a esta grandiosa potencia que el hombre ha podido liberarse del medio ambiente, del espacio y hasta clel tiempo. Para el pensamiento no hay vallas, no hay distancias, no hay épocas. Todo lo abarca en la unidad de su po- 
der ilimitado. Es gracias a este poder creador, gracias a esta cualidad divina, que al lado del edificio simétrico y maravillosamente racional de las matemáticas, puede existir la locura de la poesía dadaista. Ambas objetivaciones de la cultura tienen una fuente exclusiva y única: el pensamiento.

La posibilidad de la esfera del conocimiento, presenta una estructura bastante complicada. Por ello sólo trataremos de la posibilidad del conocimiento racional, que es el aspecto más importante del conocimiento considerado en su generalidad.

El conocimiento racional es posible cuando cumple todas las anteriores condiciones. Tiene, como se ve, la posibilidad irreal más complicada y por lo tanto su existencia sólo se puede establecer trabajosamente. Por eso llegar al conocimiento racional de cualquier objeto o strupo de objetos es una de las actividades humanas que mayores esfuerzos cuesta y más grandes sacrificios exige.

En primer lugàb el cônocimiento racional se constituye a base del pensamiento.ue manera que una proposición sin senticlo no puede jamás pretender ser vínculo de conocimiento racional. Pero además de ser pensamiento, el conocimiento racional debe de ser conocimiento lógico (de allí su nombre de racional). De manera que un pensamiento contradictorin no puede ser jamás un conocimiento racional. Por último, todo conocimiento está orientado hacia determinados objetos, y todo objeto tiene una estructura ideal, enclavada dentro de un sistema de composibilidad. No basta que un pensamiento sea lógico para que sea conocimiento racional, debe además de estar estructurado en tal forma que no contradiga a la estructura ideal del objeto conocido. 
Todas estas condiciones son prerequisitos del conocimiento racional. Pero falta aún la condición fundamental para que haya conocimiento: debe de haber adecuación con las leyes apriori del conocimiento (Kant), es decir que el pensamiento debe de estructurarse según ciertas reglas y principios (categorías). Sólo en este caso es posible ia constitución del conocimiento racional, o sea del conocimiento antiori, y el cumnlimiento de esta condición es el último nelสaño que hay que subir para que pueda determinarse st1 existencia.

La diferencia esencial entre la posibilictad irreal y la real es la diferencia de amplitud. Ta nosibilidad irreal, con diversas ọraduaciones, es amplia. Es fácil que un ente cualGuiera existe irrealmente. Es más fácil aue exista intencionalmente, a que exista lómicamente. y es raás fácil que exista lógicamente a que exista irleal $n$ cognoscitivamente. Pero de todas maneras esta existencia es amplia y fácil de comprobar.

En cambiglès sumamente dificịt Que un ente exista realmente, es decir dentro decun tiempo (y a veces dentro de un espacio) determinado.

La posibilidad real está formada por dos clases de conriciones: condiciones irreales v condiciones reales. Las condiciones irreales son condiciones lógicas y condiciones ideales. Un ente contradictorio consigo mismo no puede existir. Un ente incomposible con los principios cue estructuran a nuestro universo real tampoco puede existir. Jamás se puede dar en la realidad un triáng̣tulo redondo. Y tampoco se puede dar en la tierra y en sus proximidades un triángulo cuya suma de ángulos internos no sea igual a $180^{\circ}$ grados, porque nuestro planeta y sus proximidades (sistema solar) están organizados según los principios de la geome- 
tría euclideana. Todo ente real, debe de tener caracteres genéricos que permitan agruparlo junto a otros entes de la misma clase. Es decir que todo ente real tiene esencia. Pero la esencia es un ente ideal, por lo tanto ningún ente real puede existir si no se han cumplido anteriormente determinadas condiciones de existencia ideal, es decir las condiciones de existencia de su esencia.

Las condiciones de la existencia intencional y cognositiva no son necesarias para la existencia de un ente real. Un ente puede existir perfectamente dentro del tiempo sin que tenga para ello que ser pensado o conocido. Si no se acepta esto se cae en el idealismo con todos sus prejuicios $y$ sus incongruencias, y se contradice los datos inmediatos (iel analisis.

Aciemas de las condiciones irreales, son necesarias condiciones reales, para que un ente real sea posidie. Estas condiciones torman "complejos" o "constelaciones" de tactores convergentes. Basta que taite uno solo de ellos para que el ente sea "realmente emposible. Qtosascomplejos de condiciones reales van de lo mirseshimper aelo mas complicado contorme se avanza en los estraios de la realidad. „En el estrato de lo tisico, se reducen a complejos de causas y efectos (en el sentido de las crencras tricicas actuales, no en el sentido helénico). En el estrato de lo biologico los comprejos estan construídos por una convergencia de causas y efectos y de disposiciones y tendencias. Lis este aspecto de la posibilidact biologica, que como se ve, (tomando en cuenta las condiciones irreales y las causales), es infinitamente pequeño y estrecho, el que Aristóteles, y baju su influencia, todos los filósofos medioevales, quisieron er1gir en raiz y meollo de la posibilidad considerada en su aspecto más general. 
En el estrato de lo anímico entran, además de todos los factores considerados, factores estrictamente teleológicos, es decir volitivos, que por estar íntimamente unidos con el fenómeno de la libertad complican extraordinariamente el asunto. En el estrato de lo espiritual, la situación es tan complicada, y los estudios que se han hecho sobre la posibilidad de los entes espirituales, es decir sobre las condiciones de su existencia son tan incipientes que nada nos atrevemos a decir. Unicamente se puede afirmar que además de incluir a todas las anteriores condiciones (de los tres estratos) incluye además condiciones de valor. Lo espiritual puede únicamente constituirse cuando se presentan determinadas conexiones valorativas, es decir cuando se efectúan relaciones entre determinada entidad anímica y cierta estructura axiológica.

Demás está insistir sobre la enorme complejidad de las condiciones que constituyen la posibilidad de los entes reales. Considerese la posibindad real mas simple: la posibilidad física. Un objeto es fisicamente posible, cuando además de 10 o sèr contradictoríoctonsigo mismo, ni con el sistema de composibilidad al que pertenece su esencia, es producto de millones y millones de causas, de efectos, que a su vez son causas, y que se suceden no únicamente en forma lineal sino radial. Si se supone que el mundo ha tenido un origen en el tiempo (cosa que no se puede determinar cientificamente) el número de causas y efectos necesarios para que exista un objeto actual, es inmenso pero no infinito. Si se supone que el mundo no ha tenido origen, (cosa que tampoco tiene ningún valor científico) entonces hay que aceptar que el número de causas y efectos que constituyen la posibilidad del objeto es infinito.

Pongámosnos en el caso más fácil: el mundo tiene un origen, $y$ consideremos un ente real cualquiera: un aeroli- 
to que acaba de caer en el centro de China. ¿Cómo es posible esta caída?. Pues porque se han cumplido una serie de condiciones. .En primer lugar, tiene que haber existido un cuerpo celeste en el cual se haya originado el aerolito. La combinación de las fuerzas centrífuga y centrípeta permite que el bólido celeste salga despedido por los negros y vacíos espacios interplanetarios. Pero esto no es suficiente. Es necesario además que debido a la acción de las fuerzas gravitacionales, la tierra esté en determinado lugar del espacio con relación al sol. Es necesario que la tierra esté en determinado momento de su rotación, pues si hubiera estado en otro, el aerolito, en lugar de haber caído en China hubiera caído por ejem. en Turquía. Es imprescindible además que la materia de que esté hecho el bólido tenga determinada resistencia al roce de la atmósfera, pues de otra manera el bólido podría entrar en incandecencia y consumirse por completo como la mayor parte de los bólidos. $\mathrm{Y}$ entonces jamás habría llegado a lugar alguno de la tierra. Además es necesario que el cuerpo celeste de donde proviene esté constituído de tal o cuab nahera, pues sinó nádà podría desprenderse de él. Y para ello es menester $q u e$ dicho cuerpo haya sufrido determinada evolución geológica, evolución cuya existencia presupone una serie inconmensurable de causas y. efectos. Cataratas de causas y efectos, enjambre de condiciones, cruzamiento y entrecruzamiento de factores, convergencia y divergencia de determinaciones. Así es la posibilidad de lo real, complicada y múltiple como los granos de arena y las ondas del mar. Y basta que una sóla, nada más que una sóla de estas condiciones esté ausente para que el fenómeno real no pueda producirse, es decir para que no sea posible, para que no pueda existir. Si el bólido penetra en la estratoesfera unos segundos más tarde, ya no caerá en el centro de China, sino en otra parte, más ha- 
cia el oeste. Y por lo tanto no será posible que determinado aerolito, en determinado tiempo haya caído en el centro de la China. ¡Cuánto es necesario para la existencia

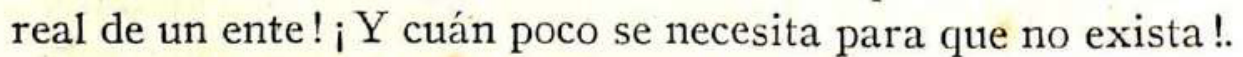
Por eso el mundo de lo real comparado con el mundo de lo irreal es como una partícula de polvo comparada con la bóveda celeste. En relación con el mundo de lo irreal, el mundo real es un enorme despilfarro die posibilidades. Casi todas las ocasiones se desperdician. Cuantos hombres son geniales por el nacimiento. Y sin embargo pasan por la vida sin dejar rastro. Eran idealmente geniales. Su esencia era la del genio. Pero faltó una pequeña condición, una condición inti$\mathrm{ma}$, sin ninguna importancia. $Y$ no pudieron ser genios. $\mathrm{Na}$ cieron en Atrica en lugar de nacer en Europa, o tuvieron una entermedad que les lesionó el cerebro, o murieron a los pocos días de nacer. Esta enorme diferencia entre la posibilidad de ser y la cle no ser, es tan importante que pueae servir para interpretar el destino del hombre y el sent1do de su estuerzo civilizador Es necesaria una infinitud de condiciones para que algo sea. Y basta una condición para que algo no sea. „El hombre lucha por el ser, por el existir. $Y$ trata de arribar a un islote de existencia en medio de un mar infinito de no ser, de inexistencia total. $Y$ en el fondo todos sus esfuerzos de progreso y de organizacion no son sino para aumentar sus posibilidades reales, para aumentar su caudal de ser y para disminuir la amenaza de no ser. Ser es dificil, no ser es fácil, he aquí la sentencia metafísica que lo amenaza desde que nace. Es la conciencia de esta posición la que lo hace un ser tan peculiar comparado con los demás seres. Los demás seres reales, son o no son. Pero no captan sus posibilidades de ser. El hombre no es, pero sabe que hubiera podido ser. Tada su vida es 
una tortura metafísica, una lucha de lo que va a dejar de ser y de lo que no puede ser, por el ser total y pleno.

El tema de la posibilidad es no sólo uno de los más profundos de toda la Filosofía, sino uno de los más difíciles. Somos los primeros en reconocer que no se puede tratar sistemáticamente en tan poco espacio como el que hemos empleado. Pero si hemos conseguido suscitar algunas reflexiones sobre su sentido y sobre los resultados teóricos rue se-derivan de ahondar en su genuina problemática, y si además hemos conseguido hacer ver, cómo a pesar de todas las dificultades y de los múltiples aspectos de irracionalidad que encierra en sí el problema de lo posible, 1q Filosofía actual ha superado con mucho a la antigua en el tratamiento del tema, permitiendo comprender otros problemas que estaban deformados por tun mal planteamiento del primero, nos daremos por satisfechos.

Francisco Miró Quesada. 